\title{
DPSK OVER INVERSE-RZ OPTICAL PULSES FOR 2-BIT PER SYMBOL TRANSMISSION
}

\author{
Tetsuya Miyazaki, Fumito Kubota \\ National Institute of Information and Communications Technology, 4-2-1 Nukuikita, Koganei, \\ 184-8795 Tokyo,Japan: tmiyazaki@nict.go.jp
}

\begin{abstract}
We successfully demonstrated superimposing DPSK over inverse-RZ optical pulses using SOA-XGM for 2-bit per symbol modulation/demodulation at 20-Gbit/s to simply double spectral efficiency. Error free operation less than $10^{-12}$ was achieved for both RZ and DPSK signals.
\end{abstract}

\section{INTRODUCTION}

Multi-state per symbol modulation format such as differential quadrature phase-shift keying (DQPSK) has been investigated to enhance spectral efficiency and tolerance to chromatic dispersion and polarization-mode-dispersion [1,2]. A combination of amplitude-shift keying (ASK) and differential phase-shift keying (DPSK) is another scheme without using complex encoder and decoder[3,4]. However precise adjustment of the extinction ratio has been required in the ASKDPSK scheme. On the other hand inverse-RZ format with more than $100 \%$ spectral efficiency was proposed without using multi-state per symbol modulation scheme[5]. In this study, we demonstrated superimposing of DPSK over inverse$\mathrm{RZ}$ as a simple solution for doubling the spectral efficiency.

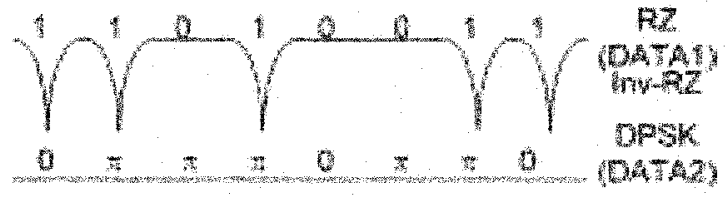

Figure 1 Timing chart of the overwriting of DPSK over inverse-RZ signal. 


\section{EXPERIMENTS AND RESULTS}

Figure 1 shows the timing chart of the overwriting of DPSK over inverse-RZ signal. Inverting of RZ optical signal allows both " 0 " and " 1 " pulses to have finite pulse energy in the time slot without adjusting the extinction ratio.

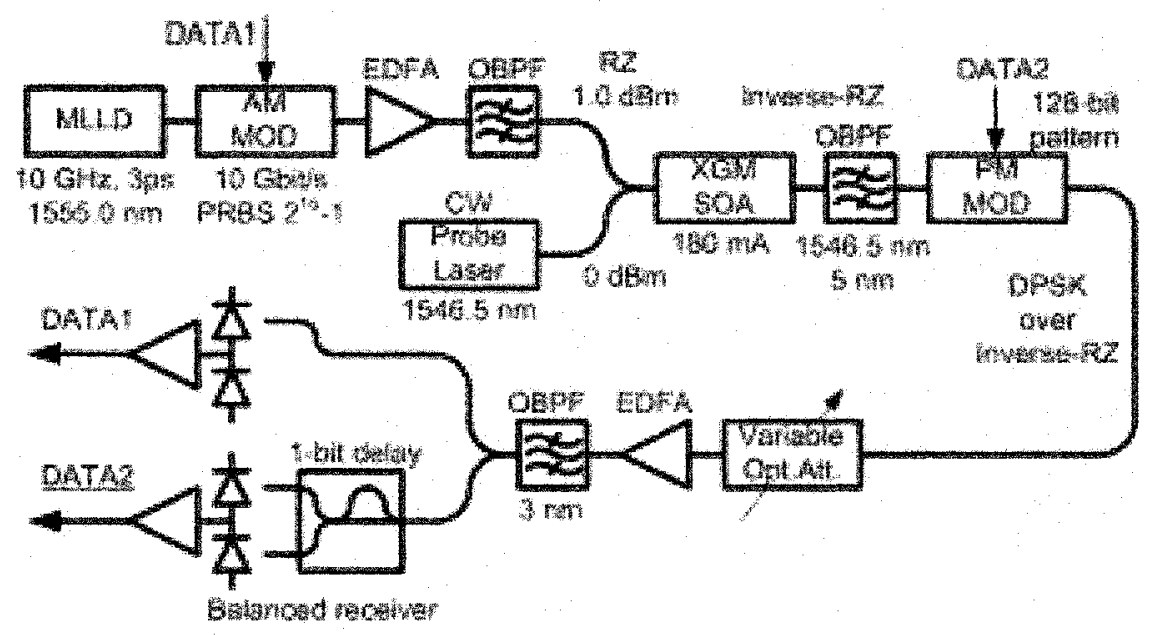

Figure 2 Experimental setup.

Figure 2 shows the setup. 10-GHz $(9.95328 \mathrm{GHz}), 3.0$ ps optical pulses from a mode-locked laser diode (MLLD, $1555 \mathrm{~nm}$ ) was modulated at $10 \mathrm{Gbit} / \mathrm{s}$ with a PRBS of $2^{15}-1$ (DATA1) by an intensity modulator for RZ modulation. For inverse- $R Z$ generation, cross-gain modulation (XGM) in a semiconductor optical amplifier (SOA, biased at $180 \mathrm{~mA}$ ) was adopted by injecting continuous wave $(\mathrm{CW})$ probe $(1546.5 \mathrm{~nm}, 4.0 \mathrm{dBm})$ and the $\mathrm{RZ}$ pulses $(-1 \mathrm{dBm})$. Previously reported technique for inverse- $R Z$ generation in electrical domain using push-pull type Mach-Zehnder modulator[5] is also applicable with an encoder to compensate alternative phase inversion. Optical band-pass filter (OBPF, $5 \mathrm{~nm}$ ) was employed to select converted inverse-RZ optical pulses. Then DPSK modulation with 128-bit pattern length (DATA2) was superimposed. In the receiver side, optical preamplifier (EDFA) followed by an OBPF ( $3 \mathrm{~nm}$ ) and a balanced receiver was used for both RZ and DPSK detection. Single-end direct detection was used to convert polarity from inverse- $R Z$ into $R Z$ pulses. We set the expected differential data pattern to an error detector.

Figure 3 shows optical waveform of DPSK with inverse-RZ measured at transmitter output (Fig. 3(a)), and detected eye diagrams of DATA 1 RZ signal 
(Fig. 3(b)), and DATA 2 DPSK signal (Fig. 3(c)). Thanks to the pulse inversion, clear eye opening was obtained in detected DPSK signal (Fig. $3(\mathrm{C})$ ). There was no prominent eye opening degradation in RZ detection by superimposing DPSK modulation (Fig.3 (b)).

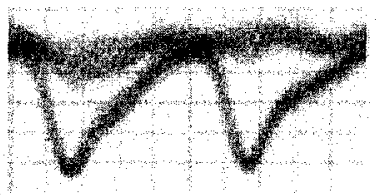

(a)transmitter output

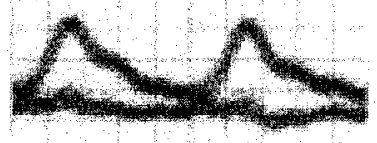

(b) DATA 1 RZ signal

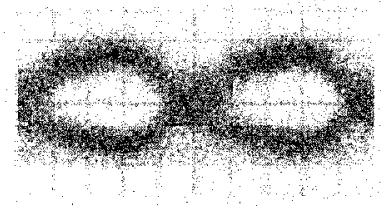

(c) DATA 2 DPSK

Figure 3 Eye diagrams ( $50 \mathrm{ps} / \mathrm{div}$.)

Figure 4 shows optical spectrum measured at the SOA output (a), at the transmitter output (b). A compact spectrum was obtained with 3-dB bandwidth of about $12 \mathrm{GHz}$ was obtained in DPSK superimposing over inverse-RZ case (Fig. 2(b)).

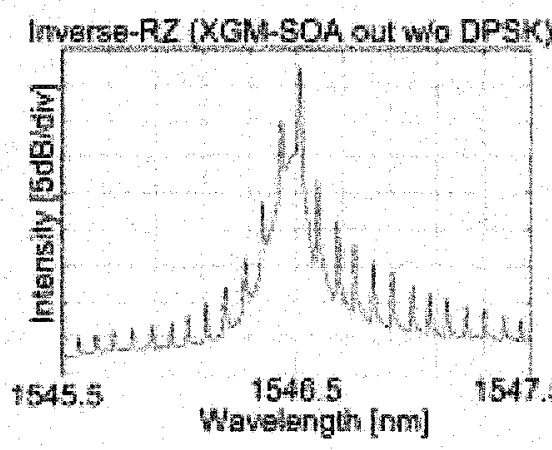

(a) SOA output

\section{DPSK superimposis oner Inverse $\mathrm{RZ}$}

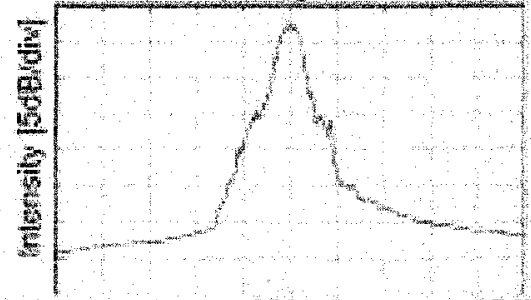

1545
1346.5

Wasternth mon

Figure 4 Optical spectrum (Span $2 \mathrm{~nm}$, resolution $0.01 \mathrm{~nm}$ )

Figure 5 shows the bit error rate (BER) characteristics for DPSK only (open circles), DATA 1: RZ (closed squares), DATA 2 : DPSK with inverse-RZ (closed circles). The received optical power was defined at the optical pre-amplifier input. It should be noted that there is only $2-\mathrm{dB}$ receiver sensitivity penalty at 10-9 in DPSK with inverse-RZ from DPSK only. There was no error floor and error free less than 10-12 was achieved for both DPSK with inverse-RZ and RZ with DPSK. 


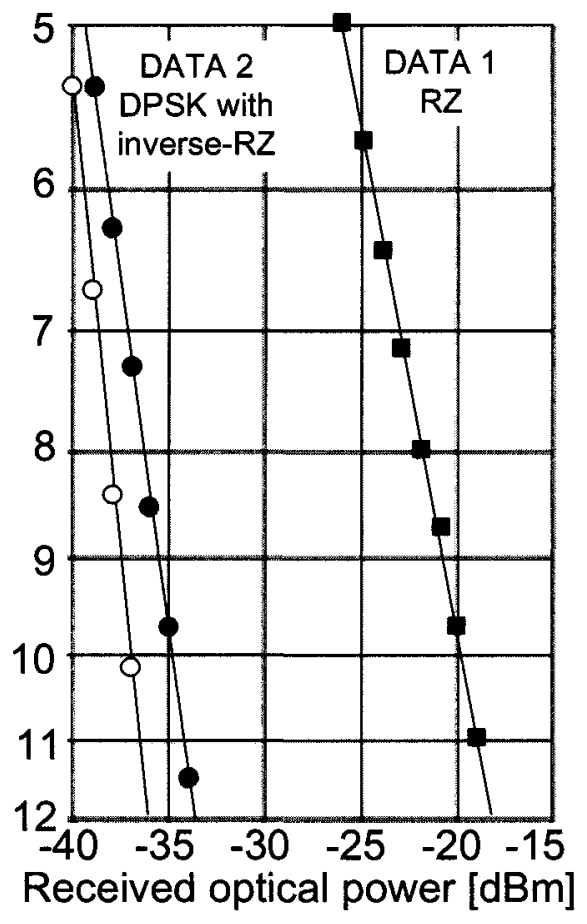

Figure 5 Bit error rate (BER) characteristics open circles : DPSK only, closed circles : DPSK with inverse-RZ, closed squares : RZ

\section{CONCLUSION}

We demonstrated DPSK over inverse-RZ format for 2-bit per symbol modulation/demodulation at $20 \mathrm{Gbit} / \mathrm{s}$ to simply double spectral efficiency. High sensitivity of DPSK data signal is preserved within $2-\mathrm{dB}$ penalty from its original sensitivity by superimposing DPSK data onto inverse- $R Z$ optical signal.

\section{ACKNOWLEDGMENTS}

The authors wish to thank Dr. Y. Matsushima for his continuous encouragement. 


\section{REFERENCES}

[1] R.A.Griffin et al, "Optical differential quadrature phase-shift key (oDQPSK) for high capacity optical transmission," OFC '02,WX6, pp.367-368, 2002.

[2] S.Hayase, et al, "Proposal of 8-state per symbol (binary ASK and QPSK) 30Gbit/s optical modulation / demodulation scheme," ECOC '03, vol.2, Tu1.6.6, pp.204-205,2003.

[3] M.Ohm et al, "Quaternary optical ASK-DPSK and receivers with direct detection," IEEE Photon. Technol. Lett.,vol.15,no.1,pp.159-161,2003.

[4] X.Liu et al., "Quaternary differential-phase amplitude-shift-keying for DWDM transmission," ECOC '03, vol.4, Th 2.6.5, pp.1010-1011, 2003.

[5] M.Ogusu et al., "Ultra-high dense WDM of Inverse-RZ signals," Technical Report of IEICE, OCS2003-105, pp.49-52, 2003. 